\title{
1 Cyanobacterial chemical production
}

3 Anna E. Case and Shota Atsumi

4

5 Department of Chemistry, University of California, Davis, CA 95616, USA

6 Correspondence should be addressed to Shota Atsumi, satsumi@ucdavis.edu, Tel:

7 +1-530-752-6595, Fax: +1-530-752-8995

8

(C) 2016. This manuscript version is made available under the Elsevier user license http://www.elsevier.com/open-access/userlicense/1.0/ 


\section{Abstract}

10 The increase in global temperatures caused by rising $\mathrm{CO}_{2}$ levels necessitates the

11 development of alternative sources of fuel and chemical. One appealing alternative

12 that has been receiving increased attention in recent years is the photosynthetic

13 conversion of atmospheric $\mathrm{CO}_{2}$ to biofuels and chemical products using genetically

14 engineered cyanobacteria. This can help to not only provide an alternate "greener"

15 source for some of the most popular petroleum based products but it can also help

16 to reduce atmospheric $\mathrm{CO}_{2}$. Utilizing cyanobacteria rather than plants allows for

17 reduced land requirements and reduces competition with food crops. This review

18 discusses advancements in the field since 2012 with a particular emphasis on

19 production of hydrocarbons.

20

21 Keywords:

22 Cyanobacteria, Photosynthesis, Metabolic Engineering, Synthetic Biology 23 


\section{1. Introduction}

25 Many products used in daily life, from gasoline for cars to nylon and plastics,

26 are derived from fossil fuels. In order to meet current energy and climate change

27 challenges we must develop alternatives to fossil fuels as sources of energy and

28 chemical production. When searching for an alternative to fossil fuels there are

29 several important factors to consider: the efficiency of chemical production, cost,

30 environmental impacts, and resources required (Keasling, 2012; Nielsen et al.,

31 2014).

32 One promising avenue currently being explored is microbial chemical

33 production. A great deal of work has been done to establish biofuel production in

34 well known fermentative organisms such as yeast and Escherichia coli (Gronenberg

35 et al., 2013; Jensen and Keasling, 2014; Rabinovitch-Deere et al., 2013). While this

36 method of chemical production is a significant improvement over traditional fossil

37 fuels, production in these host strains requires the addition of a carbon source, often

38 sugar. This carbon source comes from plants, and therefore takes land and

39 resources away from food crops, driving up the price of production (Tirado et al.,

40 2010). Waste from plant crops in the form of lignocellulosics has been explored as a

41 carbon source in these systems (Tilman et al., 2009). While advantageous in

42 eliminating the food versus fuel land use dilemma, it is often difficult to break down

43 these waste products into a usable form. Enzymes or high temperature and pressure

44 conditions are often required, which adds to the cost of production (Sanderson, 45 2011). 
Photosynthetic microorganisms offer an appealing alternative to traditional

47 production hosts (Berla et al., 2013; Nozzi et al., 2013). With the use of a

48 photosynthetic host it is possible to eliminate the need for added carbon and instead

49 fix atmospheric $\mathrm{CO}_{2}$ (Desai and Atsumi, 2013; Gronenberg et al., 2013). This also

50 provides the added benefit of combating some of the causes of global climate change

51 by the reduction of greenhouse gases. Without the need for added carbon,

52 photosynthetic hosts such as algae and cyanobacteria do not require arable lands

53 and could be grown in desert areas where they would not compete for land with

54 food crops.

55 Several different cyanobacterial host species have been investigated for use

56 in chemical production (Ducat et al., 2011; Oliver and Atsumi, 2014). A number of

57 cyanobacterial strains have been shown to be amenable to genetic manipulation and

58 the metabolic engineering tools available for such strains are increasing as interest

59 in engineering cyanobacteria grows. Chemical production has already been

60 demonstrated in cyanobacteria. In our previous review (Machado and Atsumi,

61 2012), we discussed some examples for cyanobacterial chemical production

62 including isobutyraldehyde (Atsumi et al., 2009), isobutanol (Atsumi et al., 2009), 1-

63 butanol (Lan and Liao, 2011), isoprene (Lindberg et al., 2010) and fatty acids (Liu et

64 al., 2011). This shows the promise of cyanobacterial chemical production for wide

65 scale use. However, there are several challenges to overcome before the goal of

66 large-scale chemical production from cyanobacteria can be realized (Nozzi et al.,

67 2013). These challenges include establishing tight control of gene expression,

68 improving titers and removing bottlenecks, and expanding beyond established 
69 pathways (Boyle and Silver, 2012; Camsund and Lindblad, 2014; Marcheschi et al.,

70 2013). This review discusses the progress made since 2012 (Machado and Atsumi,

71 2012) towards overcoming these obstacles with a focus on hydrocarbon production

72 (Table 1).

73

74 2. New Production Pathways in Cyanobacteria

75 One bottleneck in the broad use of cyanobacterial chemical production is the

76 limited number of chemicals that can be produced. In recent years a great deal of

77 work has been done to broaden the diversity of these chemicals and expand on the

78 utility of cyanobacteria (Table 1).

79

$80 \quad 2.1$ Alcohols and diols

81 A wide variety of pathways for the production of industrially important

82 chemicals have been developed in recent years. A prominent area of chemical

83 production in cyanobacteria is the production of alcohols and diols. Alcohols and

84 diols are both popular for use as biofuel candidates. Alcohols that have already been

85 produced in engineered cyanobacteria include 1-butanol and isobutanol (Atsumi et

86 al., 2009; Lan and Liao, 2011).

87 As mentioned in our previous review (Machado and Atsumi, 2012),

88 production of isobutanol is reliant on the valine biosynthesis pathway (Atsumi et al.,

89 2009). The intermediate 2-ketoisovalerate is funneled away from valine production

90 and redirected to isobutanol through the activities of the heterologous enzymes 2-

91 ketoacid decarboxylase and alcohol dehydrogenase (Figure 1). Similar approaches 
92 have been applied to produce 2-methyl-1-butnaol in cyanobacteria (Shen and Liao,

93 2012). 2-Methyl-1-butanol is derived from isoleucine biosynthesis and instead

94 utilizes the intermediate 2-keto-3-methylvalerate (Figure 1). A 2-ketoacid

95 decarboxylase and alcohol dehydrogenase along with the citramalate pathway were

96 utilized to produce 2-methyl-1-butanol in Synechococcus elongatus PCC7942 (7942)

97 with a final titer of $200 \mathrm{mg} / \mathrm{L}$ (Shen and Liao, 2012).

98 Isopropanol is of great interest because it can be readily converted into

99 propylene and then to polypropylene, an important industrial material (Kusakabe et

100 al., 2013). The addition of the isopropanol pathway (Figure 2) from Clostridium (an

101 acetyl transferase, acetyl-CoA acetyl transferase, acetoacetate decarboxylase, and a

102 secondary alcohol dehydrogenase) in 7942 produced $27 \mathrm{mg} / \mathrm{L}$ after 9 days

103 (Kusakabe et al., 2013). Building on this work by optimization of light conditions

104 and buffering of the growth media helped to achieve an increase in production from

$10527 \mathrm{mg} / \mathrm{L}$ to $146 \mathrm{mg} / \mathrm{L}$ (Hirokawa et al., 2015).

106 Our lab has demonstrated that the production of diols is also possible in

107 cyanobacteria, such as 2,3-butanediol (23BDO) (Oliver et al., 2013). Starting from

108 pyruvate, 23BDO production can be achieved through the addition of three

109 enzymes, acetolactate synthase, acetolactate decarboxylase, and an alcohol

110 dehydrogenase (Figure 1). Acetolactate synthase catalyzes the condensation of two

111 pyruvate molecules into one acetolactate, which is then decarboxylated to acetoin

112 by acetolactate decarboxylase. The final step is the reduction of acetoin to 23BDO

113 by a secondary alcohol dehydrogenase. We demonstrated production in 7942 and

114 final titers reached $2.4 \mathrm{~g} / \mathrm{L}$ after 20 days (Oliver et al., 2013). Similarly, 
115 Synechocystis sp. PCC 6803 (6803) was engineered to produce 23BDO by another

116 group, with a final titer of $430 \mathrm{mg} / \mathrm{L}$ (Savakis et al., 2013).

117 Cyanobacteria's natural propensity to produce fatty alcohols (Figure 2) has

118 been improved by expression of a non-native fatty-acyl coA reductase gene and the

119 deactivation of the native acyl-ACP reductase and aldehyde deformylating

120 oxygenase (Yao et al., 2014). The modifications improved fatty alcohol production

121 to $2.9 \mathrm{mg} / \mathrm{g}$ dry cell weight (DCW), up from the previously reported $0.8 \mathrm{mg} / \mathrm{g}$ DCW

122 (Yao et al., 2014).

123

1242.2 Hydroxy acids

125 The global market for plastics is expected to exceed 654 billion dollars by the

126 year 2020 (James, 2014), and plastic are currently produced primarily from

127 petroleum sources. Therefore, there is significant incentive to move away from

128 petroleum sources for the production of plastics and polymers. Toward that end the

129 production of 3 different plastic precursor molecules in cyanobacteria have been

130 established (Figure 2) (Lan et al., 2015; Wang et al., 2013a; Zhang et al., 2015). First,

131 3-hydroxybutyrate (3HB) was produced in 6803. 3HB production had been

132 previously demonstrated in E. coli by the catalysis of two enzymatic steps (Gao et al.,

133 2002). The first step requires the condensation of two acetyl-CoA molecules by a

134 thiolase to make acetoacetyl-CoA. This is followed by the reduction of acetoacetyl-

135 CoA by an acetoacetyl-CoA reductase leading to formation of 3HB. Titers reached

$136533 \mathrm{mg} / \mathrm{L}$ after 21 days (Wang et al., 2013b). Second, two different routes to

137 produce 3-hydroxypropanoic acid (3HP) in 7942 have been explored (Lan et al., 
138 2015). One pathway is malonyl-CoA dependent and produced 3HP at a maximal

139 titer of $665 \mathrm{mg} / \mathrm{L}$. The second pathway is $\beta$-alanine dependent and achieved 186

$140 \mathrm{mg} / \mathrm{L}$ 3HP (Lan et al., 2015). Last, poly-3-hydroxybutyrate (P3HB) and poly-3-

141 hydroxybutyrate-co-4-hydroxybutyrate (P3H4HB) were produced in Synechococcus

142 sp. PCC7002 (7002) through the introduction of a three or six-step pathway,

143 respectively, from Chlorogloeopsis fritschii PCC 9212 (Zhang and Bryant, 2015). The

144 gene cluster $p h a A B E C$, which encodes for an acetyl-CoA transferase, an acetyl-CoA

145 reductase and a poly(R)-hydroxy alkanoic acid synthase, was introduced on a high

146 copy plasmid (pAQ1) to convert acetyl-CoA to P3HB. Three additional enzymes, 4-

147 hydroxybutyryl-CoA transferase, 4-hydroxybutyrate dehydrogenase, and 2-

148 oxoglutarate decarboxylase imparted production of the co-polymer P3H4HB. The

149 precursor to the co-polymer, 4-hydroxybutyrate (P4HB), was produced from

150 succinic semialdehyde via the citric acid cycle. Yields achieved were $5 \%$ total cell

151 dry weight P3HB and 4.5\% of the copolymer P3H4HB (Zhang et al., 2015).

$153 \quad 2.3$ Alkanes

154 Production of a short chain alkene, ethylene, is also crucial to reduce 155 dependency on petrochemical feedstocks. Ethylene is the most widely produced

156 petrochemical feedstock on earth, and its production is the largest $\mathrm{CO}_{2}$ emitting

157 process in the chemical industry (McCoy et al., 2006). An engineered 6803 has been

158 shown to convert $\mathrm{CO}_{2}$ to ethylene (Figure 2) (Ungerer et al., 2012). This was

159 accomplished by first installing an ethylene forming enzyme (EFE) from

160 Pseudomonas syringae pv. phaseolicola (Ungerer et al., 2012). The stability of the 
161 enzyme was improved through site directed mutagenesis to achieve a final

162 production titer of $5.7 \mathrm{~mL} / \mathrm{L}$ culture/h (Ungerer et al., 2012). Ethylene production in

1636803 was also achieved through the installation of a His-tagged EFE to a rate of 0.2

$164 \mathrm{~mL} / \mathrm{L}$ culture/h. (Guerrero et al., 2012).

165 Longer chain alkanes are promising biofuel candidates (Lennen and Pfleger,

166 2013). Production of the long chain alkane heptadecane has been achieved in the

167 marine cyanobacterium Synechococcus sp. NKBG15041c (Figure 2) (Yoshino et al.,

168 2015). There are two pathways commonly seen in cyanobacteria for alkane

169 production, an acyl-acyl-carrier protein (ACP) with a reductase/aldehyde-

170 deformylating oxygenase pathway (AAR/ADO) and an $\propto$-olefin synthase pathway

171 (OLS). The two pathways had never been co-expressed in the same organism

172 (Yoshino et al., 2015). The strain was shown to naturally contain the OLS pathway,

173 resulting in the production of nonadecadiene and 1-nonadecene. The ACP,

174 AAR/ADO pathway was added to the strain to facilitate production of heptadecane.

175 The highest production level of heptadecane achieved was $4 \mu \mathrm{g} / \mathrm{g}$ DCW,

176 nonadecadiene and 1-nonadecene represented the majority of the alkanes produced

177 in the strain but amounts were not quantified (Yoshino et al., 2015).

1792.4 Fatty acids

$180 \quad$ Free fatty acids have possible applications for use in the production of

181 biodiesels. Previously, production of fatty acids was established in E. coli and has

182 also been demonstrated in two cyanobacterial species (7942 and 6803) (Figure 2),

183 though some negative physiological effects including reduced viability, altered cell 
184 membrane composition, increased reactive oxygen species and reduced

185 photosynthetic yields had been reported (Lennen et al., 2011; Liu et al., 2011;

186 Ruffing, 2013). Moving production into 7002 showed similar titers to that in 7942

187 and $6803(>130 \mathrm{mg} / \mathrm{L})$ but with reduced physiological effects as demonstrated by an

188 increased growth rate and photosynthetic yield (Ruffing, 2014).

189

$190 \quad 2.5$ Sucrose

191 As an alternative approach to producing biofuels in cyanobacteria, another

192 strategy that has been explored is the use of cyanobacteria as a sugar feedstock for

193 biofuels production in other fermentative organisms (Du et al., 2013; Ducat et al.,

194 2012). Cyanobacteria naturally accumulate sucrose under salt-stress conditions

195 (500 mM NaCl) with the addition of $\mathrm{CscB}$, a sucrose exporter from E. coli, and the

196 deletion of ADP-glucose pyrophosphorylase $(g \lg C)$ and invertase (invA) genes to

197 redirect carbon flux away from glycogen production (Figure 3). The combined

198 modifications resulted in a maximum sucrose productivity of $36 \mathrm{mg} / \mathrm{L} / \mathrm{h}$ (Ducat et

199 al., 2012). To expand sucrose production, three different cyanobacterial species

200 (7942, 6803, and Anabaena sp. PCC 7120 (7120)) were also investigated for their

201 ability to produce sucrose (Du et al., 2013). Further genetic modifications were

202 made to these strains to increase sucrose production, including the overexpression

203 of the sucrose phosphate synthase, sucrose phosphate phosphatase, and UDP-

204 glucose pyrophosphorylase genes, and the knockdown of the glucosylglycerol

205 phosphate synthase gene. Overall, a four-fold increase in sucrose production was 
206 observed over wild type strains, with final titers of $140 \mathrm{mg} / \mathrm{L}$, and rates of 5-6

$207 \mathrm{mg} / \mathrm{L} / \mathrm{h}$ (Du et al., 2013).

208

209

2.6 Glucosylglycerol

210 Glucosylglycerol (GG) has applications in pharmaceuticals and cosmetics

211 because of it's protein stabilizing and antioxidant properties (Sawangwan et al.,

212 2010). 6803 naturally produce GG in response to osmotic stress (Figure 3) (Klahn et

213 al., 2010). This natural production was enhanced by disrupting two GG uptake

214 genes and a GG repressor gene (Tan et al., 2015). GG was produced to a titer of 250

$215 \mathrm{mg} / \mathrm{L}$ after 8 days (Tan et al., 2015).

216

2172.7 Isoprenoid

218 Squalene is a 30-carbon isoprenoid commonly used in cosmetics and

219 vaccines (Figure 4) (Englund et al., 2014). The removal of a squalene hypocene

220 cyclase in 6803 resulted in the accumulation of squalene up to $0.7 \mathrm{mg} / \mathrm{OD}_{750} / \mathrm{L}$

221 (Englund et al., 2014). Additionally, no negative growth effects were observed.

222 Another compound with pharmaceutical applications, forskolin, is a diterpenoid

223 (Englund et al., 2015). Current production of forskolin requires extraction of the

224 chemical from plants because chemical synthesis is too complex to be economically

225 viable (Pateraki et al., 2014). To achieve forskolin production in cyanobacteria, two

226 diterpene synthases, CfTPS2 and CfTPS3, from Chlorogloeopsis forskohlii were

227 introduced into 6803. Together these two enzymes catalyze the conversion of

228 geranylgeranyl diphosphate to 13R-manoyl oxide (Figure 4), a precursor to 
229 forskolin. The engineered strain produced 13R-manoyl oxide at $0.5 \mathrm{mg} / \mathrm{g}$ DCW

230 (Englund et al., 2015).

231

232 2.8 Farnesene

233 Building block chemicals that can be readily converted into other chemicals

234 are of great interest in biological production. Farnesene is commonly used as a

235 building block for a large variety of different products used in everyday life

236 (Halfmann et al., 2014). The utilization of the endogenous non-mevalonate pathway

237 in 7120 enabled the strain to produce farnesene (Figure 4). The engineered strain

238 produced $70 \mu \mathrm{g} / \mathrm{L} / \mathrm{OD} /$ day farnesene (Halfmann et al., 2014).

239

240 3. Improving production in previously established pathways

241 In addition to finding new pathways for chemical production, it will also be

242 necessary to increase titers and productivities of the chemicals that have already

243 been produced. There are three main avenues that have been explored in recent

244 years to increase target chemical production from cyanobacteria: The manipulation

245 of carbon flux, additional carbon sources, and controlling gene expression.

247 3.1 Manipulating carbon flux

248 Manipulating carbon flux has the capacity to not only increase the production

249 of the desired product but also reduce non-desired side products. One mechanism

250 to increase carbon flux is to increase production of precursors, which creates a

251 "pull" toward the desired product, and can help increase the carbon flow through 
252 the pathway. For example, isoprene production in cyanobacteria had been

253 previously demonstrated with limited productivities (Lindberg et al., 2010) so

254 attempts were made to improve isoprene production by increasing carbon

255 partitioning toward isoprene (Bentley et al., 2014). This was achieved by

256 overexpression of the mevalonic acid pathway enzymes to boost production of the

257 isoprene precursors isopentenyl-diphosphate and dimethylallyl-diphosphate. This

258 altered carbon partitioning resulted in a titer of $250 \mu \mathrm{g}$ isoprene/g DCW, which

259 represented a 2.5 fold increase (Bentley et al., 2014). Additionally, our lab has been

260 able to demonstrate increased 23BDO production through increased carbon flux to

261 23BDO (Oliver and Atsumi, 2015). The overexpression of the native genes

262 responsible for pyruvate production from the Calvin cycle resulted in increased

263 carbon flux to the desired product. Overall a 1.8-fold increase in carbon fixation rate

264 was observed, $4.3 \mathrm{mM} / \mathrm{gDCW} / \mathrm{h}$ (Oliver and Atsumi, 2015).

265 Another approach to increasing carbon flux to a desired product is through

266 the removal of alternate pathways. Glycogen represents a significant carbon storage

267 mechanism in cyanobacteria that naturally redirects carbon flow away from desired

268 products (Carrieri et al., 2010; Carrieri et al., 2012; Grundel et al., 2012). This

269 concept of rerouting carbon from glycogen storage has been applied to lactate

270 production in 6803 (Figure 1) (van der Woude et al., 2014). The pathway for

271 glycogen production was deleted from the strain, and a two-fold increase in lactate

272 production was observed (van der Woude et al., 2014). However, deletion of the

273 glycogen pathway also yields observable growth deficiencies (Jacobsen et al., 2011)

274 thought to be a result of imbalanced carbon and/or redox metabolism (Li et al., 
275 2014). To avoid this issue, the use of isobutanol production as a metabolic sink was

276 investigated (Li et al., 2014). Partial growth restoration was observed with

277 expression of the isobutanol pathway in 7942, however, that restoration was

278 thought to be limited by isobutanol production (Li et al., 2014). Carbon analysis

279 revealed that $52 \%$ of total fixed carbon was directed toward isobutanol production,

280 a 2.5 fold increase over previous results lacking the glycogen deletion (Li et al., 281 2014).

282 Increased carbon flux can also be achieved through increased efficiency of

283 the enzymes in the target chemical production pathway. Increasing the turnover

284 rate of one or more key enzymes in the pathway can cause an overall increase in

285 carbon flow through the pathway. In one such case, lactic acid production in 6803

286 (Figure 1) was improved by optimizing gene expression, increasing carbon flux and

287 eliminating competing pathways (Angermayr et al., 2014). The production was

288 further increased by optimizing lactate dehydrogenase, a key enzyme in the

289 pathway, through site directed mutagenesis. Eventually, carbon partitioning to

290 lactic acid was increased from 5\% to over 50\% (Angermayr et al., 2014).

291 One of the most well know biofuels is ethanol, but current production routes

292 are heavily reliant on corn and other food crops to supply the sugar precursors

293 (Tilman et al., 2009). As an alternative to funneling food crops toward biofuel

294 production, significant work has been done to improve the production levels of a

295 previously established pathway for the production of ethanol in cyanobacteria

296 (Figure 1) (Deng and Coleman, 1999). By balancing cofactors and enzyme activities 
297 to achieve the optimal conditions for ethanol production in 6803; the final titer

298 achieved was $2.3 \mathrm{~g} / \mathrm{L}$ (Luan et al., 2015).

299 It is also possible to alter carbon flow by altering culture conditions. In one

300 such instance, salt stress $(1.24 \mathrm{M} \mathrm{NaCl})$ increased hydrogen and ethanol production

301 in 7942 and 7002, respectively (Carrieri et al., 2010).

302

303 3. 2 Additional carbon sources

304 One of the primary advantages of using cyanobacteria for biofuel production

305 is their ability to convert atmospheric $\mathrm{CO}_{2}$ into valuable products. However,

306 manipulating cyanobacteria to allow them to utilize additional carbon sources can

307 help to boost overall production levels and increase the utility of this system.

308 We have successfully demonstrated the ability of an obligate

309 photoautotrophic cyanobacterium (7942) to utilize sugar by the addition of a sugar

310 transporter (McEwen et al., 2013). This allowed for continuous growth under

311 diurnal light conditions. The strain was able to utilize glucose, sucrose, and xylose

312 under dark conditions while still maintaining its ability to fix $\mathrm{CO}_{2}$ under light

313 conditions. A different transporter was required for utilization of each sugar. For

314 glucose, a galactose: $\mathrm{H}^{+}$symporter, encoded by galP from E. coli, showed the greatest

315 and most consistent growth improvement. The addition of two enzymes, a sucrose

316 transporter and a fructokinase ( $\csc B$ and $\csc K$ ) allowed the strain to efficiently

317 utilize sucrose for improved growth under both light and dark conditions. The gene

318 cassette $x y l E A B$ from $E$. coli imparted the ability to successfully utilize xylose for

319 growth. xylE encodes a xylose transporter from E. coli, but the expression of $x y l E$ 
320 alone was toxic to cells, with a significantly reduced growth rate compared to wild

321 type, so two additional genes for a xylose isomerase $(x y l A)$ and xylulokinase $(x y l B)$

322 were required. 7942 lacks these two enzymes responsible for initiating the

323 breakdown of xylose so all three genes were required for successful utilization of

324 xylose (McEwen et al., 2013). This work was further expanded upon by

325 demonstrating 23BDO production from high density engineered 7942 strains

326 incubated under continuous light conditions with either glucose or xylose (McEwen

327 et al., 2016). From both the glucose (galP) and xylose (xylEAB) strains, a significant

328 increase in both biomass accumulation (93\% and 179\% respectively), and 23BD0

329 production (202\% and $453 \%$ respectively) was observed. A maximum 23BD0 titer

330 of $3 \mathrm{~g} / \mathrm{L}$ after 10 days was achieved from the glucose strain (McEwen et al., 2016).

331 Interestingly, under diurnal light conditions (12h light/ 12h dark) growth was

332 slower during the dark phase but production remained constant. Under constant

333 dark conditions, sugar was required for both growth and 23BDO production. In the

334 presence of glucose, $0.16 \mathrm{~g} / \mathrm{L}$ 23BDO was produced, and in the presence of xylose

$3350.18 \mathrm{~g} / \mathrm{L} 23 \mathrm{BDO}$ was produced in $72 \mathrm{~h}$ (McEwen et al., 2016). This work expanded

336 the production potential of cyanobacteria by counteracting the issue of light

337 availability during industrial scale production.

3386803 can naturally consume glucose. Isobutanol production in 6803 has been

339 demonstrated under mixotrophic conditions (Varman et al., 2013a). The addition of

340 glucose increased the isobutanol titer from $90 \mathrm{mg} / \mathrm{L}$ to $114 \mathrm{mg} / \mathrm{L}$. Further

341 improvement came from the use of oleyl alcohol as a solvent trap to relieve toxicity

342 and increased the isobutanol titer to $298 \mathrm{mg} / \mathrm{L}$ (Varman et al., 2013a). 
344 been investigated for ethylene production in 6803 (Lee et al., 2015). Two genes for

345 xylose utilization from E. coli (xylAB) were installed into the ethylene producing

3466803 strain, and led to an overall boost in production over the non-xylose

347 consuming strain. Final ethylene titers from high density cultures (OD $730 \sim 5.3$ ),

348 where light is limiting, reached $11.3 \mathrm{~mL} / \mathrm{L}$ culture, and a rate of $0.9 \mathrm{~mL} / \mathrm{L}$ culture $/ \mathrm{h}$.

349 While the use of sugar as an additional carbon source is very appealing,

350 another alternate that is arguably even more desirable are carbon sources found in

351 waste water such as acetate. Imparting the ability to utilize acetate would not only

352 bolster target chemical production but could also help remove contaminants from

353 waste streams. A strain of 6803 has been developed to produce D-lactic acid by

354 simultaneous use of acetate and $\mathrm{CO}_{2}$ (Varman et al., 2013c). Moderate

355 improvements to production were achieved through codon optimization of the ldh

356 gene (from Bacillus subtilis) and installation of a soluble transhydrogenase gene sth

357 (from Pseudomonas aeruginosa). The sth gene was installed to increase the NADH

358 pool and relieve it as a bottleneck. D-lactic acid production was further enhanced by

359 the addition of acetate to the cultures, though $\mathrm{C}^{13}$ analysis revealed that acetate

360 primarily contributed to biomass and not lactic acid production. The final D-lactic

361 acid titer achieved was $1.1 \mathrm{~g} / \mathrm{L}$ without the addition of acetate and $2.2 \mathrm{~g} / \mathrm{L}$ with the

362 addition of acetate (Varman et al., 2013c).

363

3643.3 Controlling gene expression 
365 A key factor in achieving stable gene expression in cyanobacteria is the

366 identification and development of sites in the genome for integration of exogenous

367 genes via homologous recombination into either the chromosome or endogenous

368 plasmids. A platform for high-level gene expression in 7002 has been established

369 (Xu et al., 2011). Six endogenous plasmids were adapted for the expression of non-

370 native genes. Non-native genes were successfully introduced and expressed

371 without disturbing essential genes (integration points called "neutral sites" (Xu et

372 al., 2011)). These plasmids serve as an expression platform for further exploration

373 of chemical synthesis in 7002 , and add to the metabolic engineering tools available

374 (Xu et al., 2011).

375 In another study, new neutral sites were identified that increase the sites for

376 transformation of 6803 (Ng et al., 2015). From transcriptome sequencing, two new

377 sites that do not express transcripts and thus would not disturb natural cell

378 functions were identified. One site was on the chromosome and the other on an

379 endogenous plasmid. The $y f p$ gene encoding a yellow fluorescence protein was

380 incorporated in both sites, and the site on the plasmid showed 14-fold higher

381 expression than the site on the chromosome (Ng et al., 2015).

382 It is essential to be able to control gene expression in an inducible manner.

383 Achieving high levels of production often relies upon being able to grow cells to an

384 acceptable density prior to induction, limiting growth deficiencies caused by

385 chemical production. Several different approaches for gene expression control have

386 been investigated at both the transcriptional and translational level. A theophylline

387 dependent riboswitch (Topp et al., 2010), which constitutes translational control of 
388 gene expression, was utilized to control gene expression in 7942 (Nakahira et al.,

389 2013). The best riboswitch construct demonstrated 190 -fold induction with a

390 theophylline range from 0 to $2 \mathrm{mM}$. Riboswitches can offer an additional layer of

391 gene control for production constructs (Nakahira et al., 2013) or can stand on their

392 own to control gene expression. A series of riboregulators, a form of post-

393 transcriptional gene control composed of two parts, a cis-prepressed mRNA

394 (crRNA) and a trans-activating RNA (taRNA), (Isaacs et al., 2004) were constructed

395 in 6803 (Abe et al., 2014). The crRNA contains the ribosomal binding site and forms

396 a stem-loop structure, preventing translational initiation, while the taRNA is able to

397 bind to the internal stem-loop sequence and promote gene expression. This system

398 was tested in 6803 using green fluorescent protein as a reporter, and proved

399 effective at controlling gene expression with a 13-fold increase in gfp expression

400 upon induction (Abe et al., 2014). No growth detriment was observed. A

401 riboregulator was also adapted for use in 7002 (Zess et al., 2016). When used

402 together a 59\% attenuation of target genes was observed (Zess et al., 2016).

403 At the transcriptional level, promoter systems have been adapted from other

404 host organisms and optimized for use in cyanobacteria (Markley et al., 2015). For

405 instance, E. coli IPTG inducible promoters such as pLlac01 (Lutz and Bujard, 1997)

406 and pTrc (Amann et al., 1988) have been optimized for controlling gene expression

407 in 7002 (Markley et al., 2015). The best promoter system, termed cLac143, showed

408 a 48-fold dynamic range (Markley et al., 2015).

409 A TetR-regulated (anhydrotetracycline [aTc]-dependent) promoter has been

410 developed in 6803 (Huang and Lindblad, 2013). The promoter provided a wide 
411 range of transcriptional regulation with 239-fold induction under dark conditions

412 and 290-fold induction under light conditions with $10 \mu \mathrm{g} / \mathrm{mL}$ aTc. Altering a few

413 base pairs between the -10 box and the transcriptional start site resulted a wide

414 range of induction (Huang and Lindblad, 2013). This system was later adapted to

415 create an aTc inducible promoter in 7002. This promoter showed a 32-fold

416 induction (Markley et al., 2015).

417 The ribosomal binding site can also play a large role in the level of gene

418 expression in an inducible system. We have attempted to improve production of

419 23BDO in 7942 by optimizing the ribosomal binding site of each of the three

420 transcripts in the 23BDO pathway (Oliver et al., 2014). There was not enough

421 information available to take a rational design approach so we utilized a

422 combinatorial approach with 4 different RBS sequences. We observed a broad

423 range in 23BDO production, from $207 \mathrm{mg} / \mathrm{L}$ in the weakest construct to $496 \mathrm{mg} / \mathrm{L}$ in

424 the strongest construct. This demonstrated the importance of regulating and

425 optimizing gene expression for achieving high production (Oliver et al., 2014).

426 To achieve dynamic expression range in 7002, an RBS library has been

427 designed and screened (Markley et al., 2015). The RBS sequence was modified

428 using an RBS library calculator (Salis et al., 2009) to create a library of 8 possible

429 RBS sequences with a predicted expression range of 213 -fold. In practice, a dynamic

430 range of 30-fold was observed for the library (Markley et al., 2015). The RBS library

431 successfully altered transcript level and increased the dynamic range of the system

432 but prediction of expression levels is still difficult. 
We have also explored other routes to improve gene expression and

434 regulation in cyanobacteria (Nozzi and Atsumi, 2015). Previous production of

435 23BDO in cyanobacteria had been established with poor control of production

436 (Oliver et al., 2013). The efficacy of the promoter pLac01, which was used for

437 expression of the 23BDO pathway, was investigated using a green fluorescent

438 protein as a reporter. Constructs lacking pLac01 revealed native constitutive

439 promoters in two of the genes in the 23BDO pathway (alsS and $a d h$ ), and gene order

440 was found to be critical for controlling gene expression. By optimizing gene order

441 we were able to show improvement in production and reduced leakiness. This work

442 added to the metabolic engineering "toolbox" for this strain by demonstrating that

443 pLac01 does control gene expression well and that gene order is critical in achieving

444 gene repression (Nozzi and Atsumi, 2015).

446 4. Conclusion

447 In the last few years a number of significant advancements have been made 448 toward engineering cyanobacteria for the production of valuable chemicals (Table

449 1). New pathways have been developed for the production of alcohols and diols,

450 alkanes, polymers, and even products with pharmaceutical applications such as GG

451 or squalene. In addition to a drastic broadening of target chemicals possible in these

452 host organisms, the field has also seen advancements in chemical production levels

453 driven by the manipulation of carbon flux, use of additional carbon sources, and

454 improved control of gene expression. Taken together the works described herein

455 represent a demonstration of the potential of cyanobacteria as a host organism. 
456 However, in order for cyanobacteria to become industrially relevant, further

457 work needs to be done to increase yields and productivities. Another challenge that

458 will need to be addressed is the gap between lab scale and industrial scale

459 production. At an industrial scale new factors come into play such as light

460 availability. Utilization of sugar and acetate can help bridge this gap but further

461 work will be required for successful scale up. Another limiting factor is the

462 availability of freshwater. Moving toward use of cyanobacterial strains with a

463 higher salt tolerance, or developing systems with lower water requirements such as

464 biofilms can aide in overcoming this challenge. The work done in recent years has

465 served to drastically improve the feasibility of using cyanobacteria for broad scale

466 chemical production but more work will still need to be done.

468 Acknowledgments

469 We would like to thank Christine A. Rabinovitch-Deere and Nicole E. Nozzi for

470 critical reading of the manuscript. This work was supported by the National Science

471 Foundation CBET-1349663. 


\section{References}

473 Abe, K., Sakai, Y., Nakashima, S., Araki, M., Yoshida, W., Sode, K., Ikebukuro, K.,

474 (2014) Design of riboregulators for control of cyanobacterial (Synechocystis) protein 475 expression. Biotechnol Lett 36, 287-294.

476 Amann, E., Ochs, B., Abel, K.-J., (1988) Tightly regulated tac promoter vectors useful 477 for the expression of unfused and fused proteins in Escherichia coli. Gene 69, 301478315.

479 Angermayr, S.A., van der Woude, A.D., Correddu, D., Vreugdenhil, A., Verrone, V., 480 Hellingwerf, K.J., (2014) Exploring metabolic engineering design principles for the 481 photosynthetic production of lactic acid by Synechocystis sp. PCC6803. Biotechnol 482 Biofuels 7, 1-15.

483 Atsumi, S., Higashide, W., Liao, J.C., (2009) Direct photosynthetic recycling of carbon 484 dioxide to isobutyraldehyde. Nat Biotechnol 27, 1177-1180.

485 Bentley, F.K., Zurbriggen, A., Melis, A., (2014) Heterologous expression of the 486 mevalonic acid pathway in cyanobacteria enhances endogenous carbon partitioning 487 to isoprene. Mol Plant 7, 71-86.

488 Berla, B.M., Saha, R., Immethun, C.M., Maranas, C.D., Moon, T.S., Pakrasi, H.B., (2013)

489 Synthetic biology of cyanobacteria: unique challenges and opportunities. Front $490 \quad$ Microbiol 4, 246.

491 Boyle, P.M., Silver, P.A., (2012) Parts plus pipes: synthetic biology approaches to 492 metabolic engineering. Metab Eng 14, 223-232.

493 Camsund, D., Lindblad, P., (2014) Engineered transcriptional systems for 494 cyanobacterial biotechnology. Front Bioeng Biotechnol 2, 40. 
495 Carrieri, D., Momot, D., Brasg, I.A., Ananyev, G., Lenz, O., Bryant, D.A., Dismukes, G.C.,

496 (2010) Boosting autofermentation rates and product yields with sodium stress

497 cycling: application to production of renewable fuels by cyanobacteria. Appl Environ 498 Microbiol 76, 6455-6462.

499 Carrieri, D., Paddock, T., Maness, P.C., Seibert, M., Yu, J.P., (2012) Photo-catalytic

500 conversion of carbon dioxide to organic acids by a recombinant cyanobacterium

501 incapable of glycogen storage. Energy Environ Sci 5, 9457-9461.

502 Deng, M.D., Coleman, J.R., (1999) Ethanol synthesis by genetic engineering in

503 cyanobacteria. Appl Environ Microbiol 65, 523-528.

504 Desai, S.H., Atsumi, S., (2013) Photosynthetic approaches to chemical biotechnology.

505 Curr Opin Biotechnol 24, 1031-1036.

506 Du, W., Liang, F., Duan, Y., Tan, X., Lu, X., (2013) Exploring the photosynthetic

507 production capacity of sucrose by cyanobacteria. Metab Eng 19, 17-25.

508 Ducat, D.C., Avelar-Rivas, J.A., Way, J.C., Silver, P.A., (2012) Rerouting carbon flux to

509 enhance photosynthetic productivity. Appl Environ Microbiol 78, 2660-2668.

510 Ducat, D.C., Way, J.C., Silver, P.A., (2011) Engineering cyanobacteria to generate

511 high-value products. Trends Biotechnol 29, 95-103.

512 Englund, E., Andersen-Ranberg, J., Miao, R., Hamberger, B., Lindberg, P., (2015)

513 Metabolic engineering of Synechocystis sp. PCC 6803 for production of the plant

514 diterpenoid manoyl oxide. ACS Synth Biol 4, 1270-1278.

515 Englund, E., Pattanaik, B., Ubhayasekera, S.J., Stensjo, K., Bergquist, J., Lindberg, P.,

516 (2014) Production of squalene in Synechocystis sp. PCC 6803. PLoS One 9, e90270. 
517 Gao, H.J., Wu, Q., Chen, G.Q., (2002) Enhanced production of D-(-)-3-hydroxybutyric

518 acid by recombinant Escherichia coli. FEMS Microbiol Lett 213, 59-65.

519 Gronenberg, L.S., Marcheschi, R.J., Liao, J.C., (2013) Next generation biofuel

520 engineering in prokaryotes. Curr Opin Chem Biol 17, 462-471.

521 Grundel, M., Scheunemann, R., Lockau, W., Zilliges, Y., (2012) Impaired glycogen

522 synthesis causes metabolic overflow reactions and affects stress responses in the

523 cyanobacterium Synechocystis sp. PCC 6803. Microbiology 158, 3032-3043.

524 Guerrero, F., Carbonell, V., Cossu, M., Correddu, D., Jones, P.R., (2012) Ethylene

525 synthesis and regulated expression of recombinant protein in Synechocystis sp. PCC

526 6803. PLoS One 7.

527 Halfmann, C., Gu, L., Gibbons, W., Zhou, R., (2014) Genetically engineering

528 cyanobacteria to convert $\mathrm{CO}_{2}$, water, and light into the long-chain hydrocarbon

529 farnesene. Appl Microbiol Biotechnol 98, 9869-9877.

530 Hirokawa, Y., Suzuki, I., Hanai, T., (2015) Optimization of isopropanol production by

531 engineered cyanobacteria with a synthetic metabolic pathway. J Biosci Bioeng 119,

$532 \quad 585-590$.

533 Huang, H.H., Lindblad, P., (2013) Wide-dynamic-range promoters engineered for

534 cyanobacteria. J Biol Eng 7, 10.

535 Isaacs, F.J., Dwyer, D.J., Ding, C., Pervouchine, D.D., Cantor, C.R., Collins, J.J., (2004)

536 Engineered riboregulators enable post-transcriptional control of gene expression.

$537 \quad$ Nat Biotechnol 22, 841-847. 
538 Jacobsen, J.H., Rosgaard, L., Sakuragi, Y., Frigaard, N.U., (2011) One-step plasmid

539 construction for generation of knock-out mutants in cyanobacteria: studies of

540 glycogen metabolism in Synechococcus sp. PCC 7002. Photosynth Res 107, 215-221.

541 James, S., (2014) Global Plastics Market, Size \& Forecast to 2020 by Grand View

542 Research, Inc.

543 Jensen, M.K., Keasling, J.D., (2014) Recent applications of synthetic biology tools for

544 yeast metabolic engineering. FEMS Yeast Res 15, 1-10.

545 Justin Ungerer, L.T., Mark Davis, Maria Ghirardi, Pin-Ching Maness and Jianping Yu,

546 (2012) Sustained photosynthetic conversion of $\mathrm{CO}_{2}$ to ethylene in recombinant

547 cyanobacterium Synechocystis 6803. Energy Enviorn Sci 5, 8998-9006.

548 Keasling, J.D., (2012) Synthetic biology and the development of tools for metabolic

549 engineering. Metab Eng 14, 189-195.

550 Klahn, S., Hohne, A., Simon, E., Hagemann, M., (2010) The gene ssl3076 encodes a

551 protein mediating the salt-induced expression of ggpS for the biosynthesis of the

552 compatible solute glucosylglycerol in Synechocystis sp. strain PCC 6803. J Bacteriol 553 192, 4403-4412.

554 Kusakabe, T., Tatsuke, T., Tsuruno, K., Hirokawa, Y., Atsumi, S., Liao, J.C., Hanai, T.,

555 (2013) Engineering a synthetic pathway in cyanobacteria for isopropanol

556 production directly from carbon dioxide and light. Metab Eng 20, 101-108.

557 Lan, E.I., Chuang, D.S., Shen, C.R., Lee, A.M., Ro, S.Y., Liao, J.C., (2015) Metabolic

558 engineering of cyanobacteria for photosynthetic 3-hydroxypropionic acid

559 production from $\mathrm{CO}_{2}$ using Synechococcus elongatus PCC 7942. Metab Eng 31, 163560170. 
561 Lan, E.I., Liao, J.C., (2011) Metabolic engineering of cyanobacteria for 1-butanol

562 production from carbon dioxide. Metab Eng 13, 353-363.

563 Lee, T.C., Xiong, W., Paddock, T., Carrieri, D., Chang, I.F., Chiu, H.F., Ungerer, J., Juo,

564 S.H., Maness, P.C., Yu, J., (2015) Engineered xylose utilization enhances bio-products

565 productivity in the cyanobacterium Synechocystis sp. PCC 6803. Metab Eng 30, 179-

566189.

567 Lennen, R.M., Kruziki, M.A., Kumar, K., Zinkel, R.A., Burnum, K.E., Lipton, M.S.,

568 Hoover, S.W., Ranatunga, D.R., Wittkopp, T.M., Marner, W.D., 2nd, Pfleger, B.F.,

569 (2011) Membrane stresses induced by overproduction of free fatty acids in

570 Escherichia coli. Appl Environ Microbiol 77, 8114-8128.

571 Lennen, R.M., Pfleger, B.F., (2013) Microbial production of fatty acid-derived fuels

572 and chemicals. Curr Opin Biotechnol 24, 1044-1053.

573 Li, X., Shen, C.R., Liao, J.C., (2014) Isobutanol production as an alternative metabolic

574 sink to rescue the growth deficiency of the glycogen mutant of Synechococcus

575 elongatus PCC 7942. Photosynth Res 120, 301-310.

576 Lindberg, P., Park, S., Melis, A., (2010) Engineering a platform for photosynthetic

577 isoprene production in cyanobacteria, using Synechocystis as the model organism.

578 Metab Eng 12, 70-79.

579 Liu, X., Sheng, J., Curtiss, R., 3rd, (2011) Fatty acid production in genetically modified

580 cyanobacteria. Proc Natl Acad Sci U S A 108, 6899-6904.

581 Luan, G., Qi, Y., Wang, M., Li, Z., Duan, Y., Tan, X., Lu, X., (2015) Combinatory strategy

582 for characterizing and understanding the ethanol synthesis pathway in

583 cyanobacteria cell factories. Biotechnol Biofuels 8, 184. 
584 Lutz, R., Bujard, H., (1997) Independent and tight regulation of transcriptional units

585 in Escherichia coli via the LacR/O, the TetR/O and AraC/I1-I2 regulatory elements.

586 Nucleic Acids Res 25, 1203-1210.

587 Machado, I.M., Atsumi, S., (2012) Cyanobacterial biofuel production. J Biotechnol

$588 \quad 162,50-56$.

589 Marcheschi, R.J., Gronenberg, L.S., Liao, J.C., (2013) Protein engineering for

590 metabolic engineering: current and next-generation tools. Biotechnol J 8, 545-555.

591 Markley, A.L., Begemann, M.B., Clarke, R.E., Gordon, G.C., Pfleger, B.F., (2015)

592 Synthetic biology toolbox for controlling gene expression in the cyanobacterium

593 Synechococcus sp. strain PCC 7002. ACS Synth Biol 4, 595-603.

594 McCoy, M., Reisch, M., Tullo, A., (2006) Production: Growth Is the Norm. Chemical \& 595 Engineering News 84, 59-68.

596 McEwen, J.T., Kanno, M., Atsumi, S., (2016) 2,3 Butanediol production in an obligate

597 photoautotrophic cyanobacterium in dark conditions via diverse sugar

598 consumption. Metab Eng 36, 28-36.

599 McEwen, J.T., Machado, I.M., Connor, M.R., Atsumi, S., (2013) Engineering

600 Synechococcus elongatus PCC 7942 for continuous growth under diurnal conditions.

601 Appl Environ Microbiol 79, 1668-1675.

602 Nakahira, Y., Ogawa, A., Asano, H., Oyama, T., Tozawa, Y., (2013) Theophylline-

603 dependent riboswitch as a novel genetic tool for strict regulation of protein

604 expression in cyanobacterium Synechococcus elongatus PCC 7942. Plant Cell Physiol $605 \quad 54,1724-1735$. 
606 Ng, A.H., Berla, B.M., Pakrasi, H.B., (2015) Fine-tuning of photoautotrophic protein

607 production by combining promoters and neutral sites in the cyanobacterium

608 Synechocystis sp. Strain PCC 6803. Appl Environ Microbiol 81, 6857-6863.

609 Nielsen, J., Fussenegger, M., Keasling, J., Lee, S.Y., Liao, J.C., Prather, K., Palsson, B.,

610 (2014) Engineering synergy in biotechnology. Nat Chem Biol 10, 319-322.

611 Nozzi, N.E., Atsumi, S., (2015) Genome engineering of the 2,3-butanediol

612 biosynthetic pathway for tight regulation in cyanobacteria. ACS Synth Biol 4, 1197-

6131204.

614 Nozzi, N.E., Oliver, J.W., Atsumi, S., (2013) Cyanobacteria as a platform for biofuel

615 production. Front Bioeng Biotechnol 1, 7.

616 Oliver, J.W., Atsumi, S., (2014) Metabolic design for cyanobacterial chemical

617 synthesis. Photosynth Res 120, 249-261.

618 Oliver, J.W., Atsumi, S., (2015) A carbon sink pathway increases carbon productivity

619 in cyanobacteria. Metab Eng 29, 106-112.

620 Oliver, J.W., Machado, I.M., Yoneda, H., Atsumi, S., (2013) Cyanobacterial conversion

621 of carbon dioxide to 2,3-butanediol. Proc Natl Acad Sci U S A 110, 1249-1254.

622 Oliver, J.W., Machado, I.M., Yoneda, H., Atsumi, S., (2014) Combinatorial optimization

623 of cyanobacterial 2,3-butanediol production. Metab Eng 22, 76-82.

624 Pateraki, I., Andersen-Ranberg, J., Hamberger, B., Heskes, A.M., Martens, H.J., Zerbe, 625 P., Bach, S.S., Moller, B.L., Bohlmann, J., Hamberger, B., (2014) Manoyl oxide (13R), 626 the biosynthetic precursor of forskolin, is synthesized in specialized root cork cells 627 in Coleus forskohlii. Plant Physiol 164, 1222-1236. 
628 Rabinovitch-Deere, C.A., Oliver, J.W., Rodriguez, G.M., Atsumi, S., (2013) Synthetic

629 biology and metabolic engineering approaches to produce biofuels. Chem Rev 113, $630 \quad 4611-4632$.

631 Ruffing, A.M., (2013) Borrowing genes from Chlamydomonas reinhardtii for free 632 fatty acid production in engineered cyanobacteria. J Appl Phycol 25, 1495-1507. 633 Ruffing, A.M., (2014) Improved Free Fatty Acid Production in Cyanobacteria with 634 Synechococcus sp. PCC 7002 as Host. Front Bioeng Biotechnol 2, 17.

635 Salis, H.M., Mirsky, E.A., Voigt, C.A., (2009) Automated design of synthetic ribosome 636 binding sites to control protein expression. Nat Biotechnol 27, 946-950.

637 Sanderson, K., (2011) Lignocellulose: A chewy problem. Nature 474, S12-14.

638 Savakis, P.E., Angermayr, S.A., Hellingwerf, K.J., (2013) Synthesis of 2,3-butanediol 639 by Synechocystis sp. PCC6803 via heterologous expression of a catabolic pathway 640 from lactic acid- and enterobacteria. Metab Eng 20, 121-130.

641 Sawangwan, T., Goedl, C., Nidetzky, B., (2010) Glucosylglycerol and

642 glucosylglycerate as enzyme stabilizers. Biotechnol J 5, 187-191.

643 Shen, C.R., Liao, J.C., (2012) Photosynthetic production of 2-methyl-1-butanol from

$644 \mathrm{CO}_{2}$ in cyanobacterium Synechococcus elongatus PCC7942 and characterization of 645 the native acetohydroxyacid synthase. Energy Environ Sci 5, 9574-9583.

646 Tan, X., Du, W., Lu, X., (2015) Photosynthetic and extracellular production of 647 glucosylglycerol by genetically engineered and gel-encapsulated cyanobacteria. Appl 648 Microbiol Biotechnol 99, 2147-2154. 
649 Tilman, D., Socolow, R., Foley, J.A., Hill, J., Larson, E., Lynd, L., Pacala, S., Reilly, J.,

650 Searchinger, T., Somerville, C., Williams, R., (2009) Energy. Beneficial biofuels--the

651 food, energy, and environment trilemma. Science 325, 270-271.

652 Tirado, M.C., Cohen, M.J., Aberman, N., Meerman, J., Thompson, B., (2010) Addressing

653 the challenges of climate change and biofuel production for food and nutrition

654 security. Food Research International 43, 1729-1744.

655 Topp, S., Reynoso, C.M., Seeliger, J.C., Goldlust, I.S., Desai, S.K., Murat, D., Shen, A.,

656 Puri, A.W., Komeili, A., Bertozzi, C.R., Scott, J.R., Gallivan, J.P., (2010) Synthetic

657 riboswitches that induce gene expression in diverse bacterial species. Appl Environ

658 Microbiol 76, 7881-7884.

659 Ungerer, J., Tao, L., Davis, M., Ghirardi, M., Maness, P.C., Yu, J.P., (2012) Sustained

660 photosynthetic conversion of $\mathrm{CO}_{2}$ to ethylene in recombinant cyanobacterium

661 Synechocystis 6803. Energy Environ Sci 5, 8998-9006.

662 van der Woude, A.D., Angermayr, S.A., Puthan Veetil, V., Osnato, A., Hellingwerf, K.J.,

663 (2014) Carbon sink removal: Increased photosynthetic production of lactic acid by

664 Synechocystis sp. PCC6803 in a glycogen storage mutant. J Biotechnol 184, 100-102.

665 Varman, A.M., Xiao, Y., Pakrasi, H.B., Tang, Y.J., (2013a) Metabolic engineering of

666 Synechocystis sp. strain PCC 6803 for isobutanol production. Appl Environ Microbiol $667 \quad 79,908-914$.

668 Varman, A.M., Yu, Y., You, L., Tang, Y.J., (2013b) Photoautotrophic production of D-

669 lactic acid in an engineered cyanobacterium. Microb Cell Fact 12.

670 Varman, A.M., Yu, Y., You, L., Tang, Y.J., (2013c) Photoautotrophic production of D-

671 lactic acid in an engineered cyanobacterium. Microb Cell Fact 12, 117-124. 
672 Wang, B., Pugh, S., Nielsen, D.R., Zhang, W., Meldrum, D.R., (2013a) Engineering

673 cyanobacteria for photosynthetic production of 3-hydroxybutyrate directly from

$674 \mathrm{CO}_{2}$. Metab Eng 16, 68-77.

675 Wang, W., Liu, X., Lu, X., (2013b) Engineering cyanobacteria to improve

676 photosynthetic production of alka(e)nes. Biotechnol Biofuels 6.

677 Xu, Y., Alvey, R.M., Byrne, P.O., Graham, J.E., Shen, G., Bryant, D.A., (2011) Expression

678 of genes in cyanobacteria: adaptation of endogenous plasmids as platforms for high-

679 level gene expression in Synechococcus sp. PCC 7002. Methods Mol Biol 684, 273-

680293.

681 Yao, L., Qi, F., Tan, X., Lu, X., (2014) Improved production of fatty alcohols in

682 cyanobacteria by metabolic engineering. Biotechnol Biofuels 7, 94 .

683 Yoshino, T., Liang, Y., Arai, D., Maeda, Y., Honda, T., Muto, M., Kakunaka, N., Tanaka,

684 T., (2015) Alkane production by the marine cyanobacterium Synechococcus sp.

685 NKBG15041c possessing the alpha-olefin biosynthesis pathway. Appl Microbiol

686 Biotechnol 99, 1521-1529.

687 Zess, E.K., Begemann, M.B., Pfleger, B.F., (2016) Construction of new synthetic

688 biology tools for the control of gene expression in the cyanobacterium

689 Synechococcus sp. strain PCC 7002. Biotechnol Bioeng 113, 424-432.

690 Zhang, S., Bryant, D.A., (2015) Biochemical validation of the glyoxylate cycle in the

691 cyanobacterium Chlorogloeopsis fritschii Strain PCC 9212. J Biol Chem 290, 14019-

69214030. 
693 Zhang, S., Liu, Y., Bryant, D.A., (2015) Metabolic engineering of Synechococcus sp.

694 PCC 7002 to produce poly-3-hydroxybutyrate and poly-3-hydroxybutyrate-co-4-

695 hydroxybutyrate. Metab Eng 32, 174-183.

696

697 
Table 1 Chemical production in cyanobacteria

\begin{tabular}{|c|c|c|c|c|}
\hline Product & $\begin{array}{l}\text { Titer/ } \\
\text { Productivity }\end{array}$ & Host & $\begin{array}{l}\text { Additional } \\
\text { carbon } \\
\text { source }\end{array}$ & Reference \\
\hline 2-methyl-1-butanol & $0.2 \mathrm{~g} / \mathrm{L}$ & 7942 & & (Shen and Liao, 2012) \\
\hline Isopropanol & $0.1 \mathrm{~g} / \mathrm{L}$ & 7942 & & (Hirokawa et al., 2015) \\
\hline 23BDO & $2.4 \mathrm{~g} / \mathrm{L}$ & 7942 & & (Oliver et al., 2013) \\
\hline 23BDO & $3 \mathrm{~g} / \mathrm{L}$ & 7942 & glucose & (McEwen et al., 2016) \\
\hline Fatty alcohols & $3 \mathrm{mg} / \mathrm{gDCW}$ & 6803 & & (Yao et al., 2014) \\
\hline Ethanol & $2.3 \mathrm{~g} / \mathrm{L}$ & 6803 & & (Luan et al., 2015) \\
\hline ЗНB & $0.5 \mathrm{~g} / \mathrm{L}$ & 6803 & & (Wang et al., 2013a) \\
\hline 3HP & $0.7 \mathrm{~g} / \mathrm{L}$ & 7942 & & (Lan et al., 2015) \\
\hline Р3НB & $5 \% \mathrm{DCW}$ & 7002 & & (Zhang et al., 2015) \\
\hline Р3H4HB & $4.5 \% \mathrm{DCW}$ & 7002 & & (Zhang et al., 2015) \\
\hline Ethylene & $5.7 \mathrm{~mL} / \mathrm{L} / \mathrm{h}$ & 6803 & & (Ungerer et al., 2012) \\
\hline Ethylene & $0.9 \mathrm{~mL} / \mathrm{L} / \mathrm{h}$ & 6803 & xylose & (Lee et al., 2015) \\
\hline Heptadecane & $4 \mu \mathrm{g} / \mathrm{gDCW}$ & $15041 c$ & & (Yoshino et al., 2015) \\
\hline Free fatty acids & $0.2 \mathrm{~g} / \mathrm{L}$ & 6803 & & (Liu et al., 2011) \\
\hline Sucrose & $0.1 \mathrm{~g} / \mathrm{L}$ & 7942 & & (Ducat et al., 2012) \\
\hline Sucrose & $0.1 \mathrm{~g} / \mathrm{L}$ & 6803 & & (Du et al., 2013) \\
\hline GG & $0.3 \mathrm{~g} / \mathrm{L}$ & 6803 & & (Tan et al., 2015) \\
\hline Squalene & $0.7 \mathrm{mg} / \mathrm{OD} / \mathrm{L}$ & 6803 & & (Englund et al., 2014) \\
\hline 13R-manoyl oxide & $0.5 \mathrm{mg} / \mathrm{gDCW}$ & 6803 & & (Englund et al., 2015) \\
\hline Farnesene & $70 \mu \mathrm{g} / \mathrm{L} / \mathrm{OD} / \mathrm{day}$ & 7120 & & (Halfmann et al., 2014) \\
\hline Isoprene & $0.3 \mathrm{mg} / \mathrm{gDCW}$ & 6803 & & (Bentley et al., 2014) \\
\hline & & & & (Angermayr et al., \\
\hline Lactic acid & $0.8 \mathrm{~g} / \mathrm{L}$ & 6803 & & 2014) \\
\hline Lactic acid & $2.2 \mathrm{~g} / \mathrm{L}$ & 6803 & acetate & (Varman et al., 2013b) \\
\hline Isobutanol & $0.3 \mathrm{~g} / \mathrm{L}$ & 6803 & glucose & (Varman et al., 2013a) \\
\hline
\end{tabular}




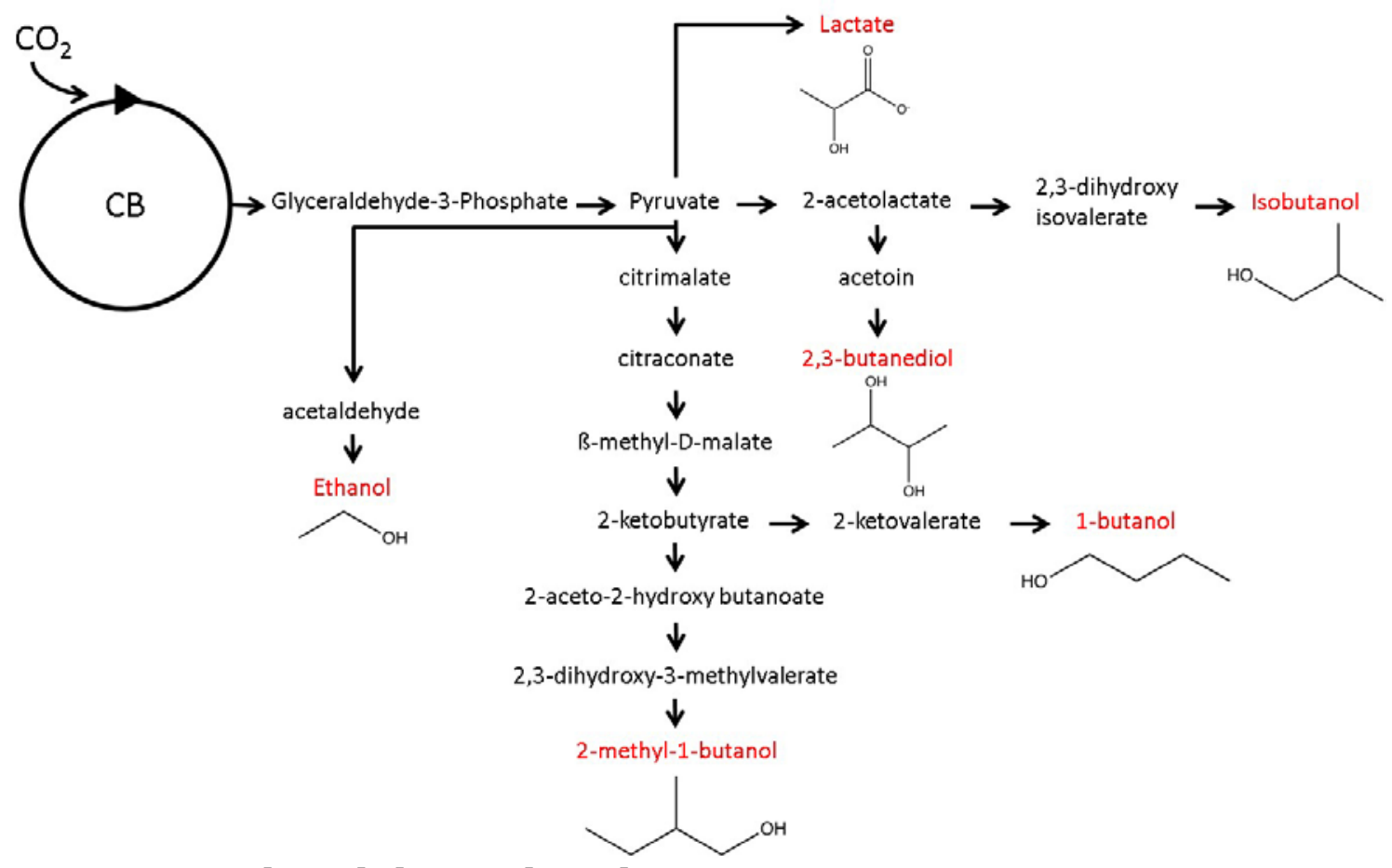

\section{Fig. 1 Pyruvate based chemical production}

703 Production of pyruvate derived alcohols and diols from atmospheric $\mathrm{CO}_{2}$ in

704 cyanobacteria. End products are highlighted in red and are shown with the chemical 705 structure. CB- Calvin-Benson cycle.

706 


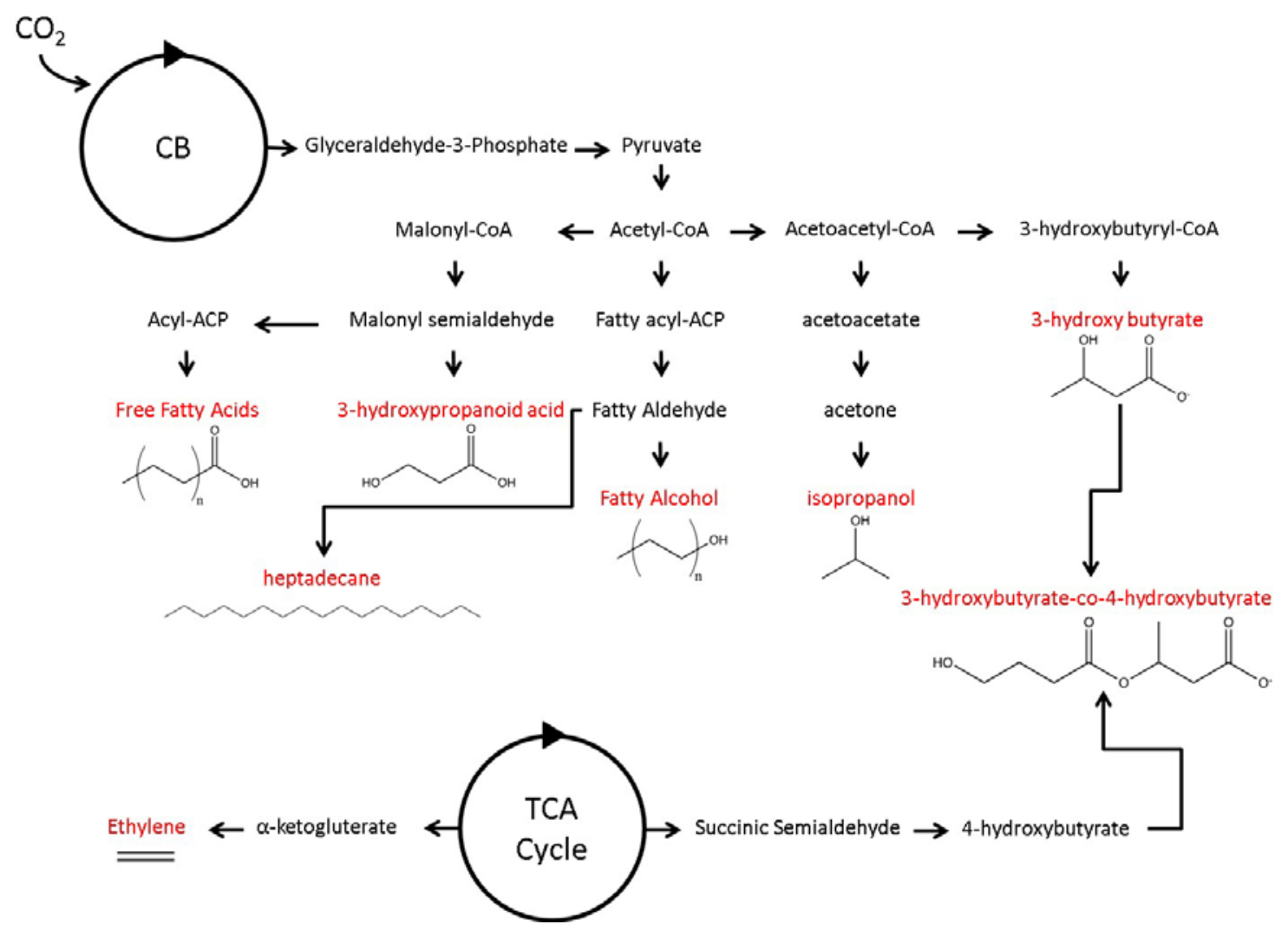

\section{Fig. 2 Production of hydroxy acids and alkanes in cyanobacteria}

709

Production of hydroxy acids and alkanes from acetyl-CoA and the tricarboxylic acid

710 cycle (TCA) cycle in cyanobacteria. End products are highlighted in red and are 
716 End products are highlighted in red and are shown with the chemical structure. 


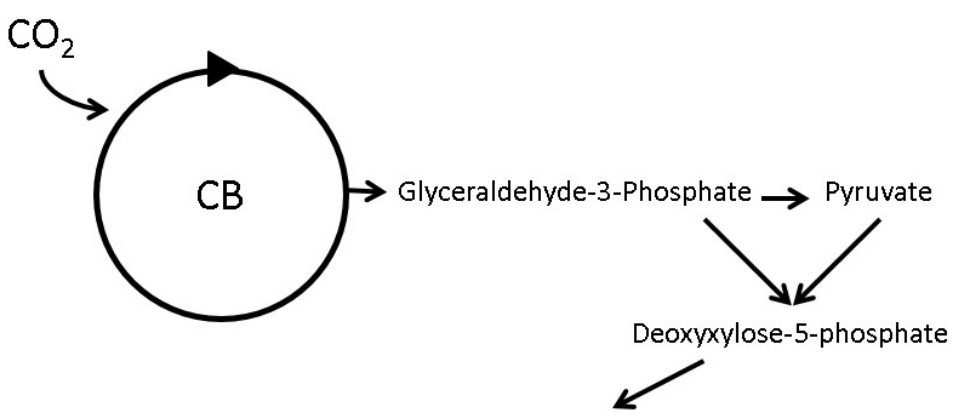

Methylerythritol-4-phosphate

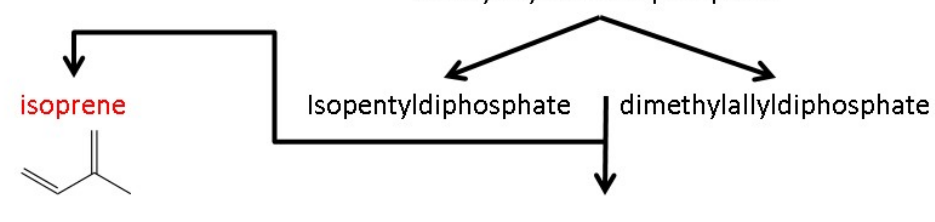

eranyl diphosphate

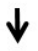

Farnesyl diphosphate

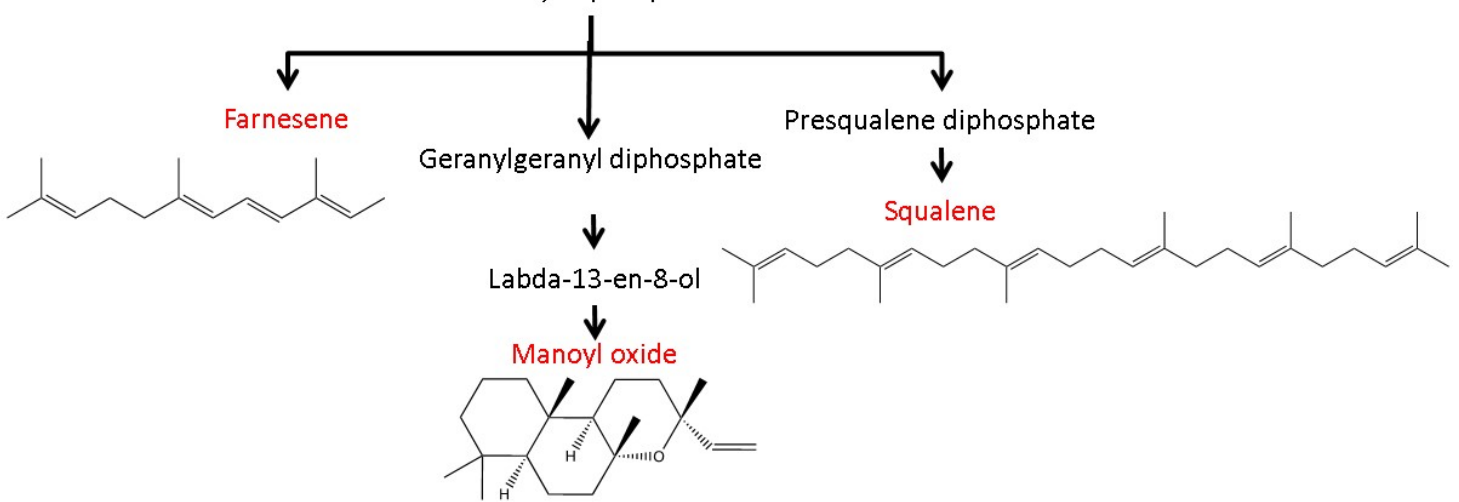

Fig. 4 Cyanobacterial production of isoprenoids

720 Production of isoprenoids from atmospheric $\mathrm{CO}_{2}$ in cyanobacteria. End products are

721

722 highlighted in red and shown with chemical structure. 